Pacific

Journal of

Mathematics

RIGIDITY OF COMPACT MINIMAL SUBMANIFOLDS IN A SPHERE

ShARIEF Deshmukh

Volume 193 No. 1

March 2000 


\section{RIGIDITY OF COMPACT MINIMAL SUBMANIFOLDS IN A SPHERE}

\section{SHARIEF DESHMUKH}

In this paper we study $n$-dimensional compact minimal submanifolds in $S^{n+p}$ with scalar curvature $S$ satisfying the pinching condition $S>n(n-2)$. We show that for $p \leq 2$ these submanifolds are totally geodesic (cf. Theorem 3.2 and Corollary 3.1). However, for codimension $p \geq 2$, we prove the result under an additional restrictions on the curvature tensor corresponding to the normal connection (cf. Theorem 3.1 and Corollary 4.1). We also show that the scalar curvature $S$ of a non-totally geodesic $n$-dimensional non-negatively curved minimal submanifold in $S^{n+p}$ with flat normal connection satisfies $n(n-p-1) \leq S \leq n(n-2)$ (cf. Theorem 4.1). Since for a compact hypersurface $M$ of $S^{n+1}$ the normal connection is flat, we use the above estimate for a scalar curvature $S$ of a non-negatively curved minimal hypersurface $M$ in $S^{n+1}$ to infer that either $M$ is totally geodesic or else it is isometric to the hypersurface $S^{m}\left(\sqrt{\frac{m}{n}}\right) \times S^{n-m}\left(\sqrt{\frac{n-m}{n}}\right)$. As a consequence this result, we conclude that the only non-negatively curved compact minimal hypersurfaces in $S^{n+1}$ which are diffeomorphic to $S^{n}$ is totally geodesic sphere.

\section{Introduction.}

Let $M$ be an $n$-dimensional compact minimal submanifold in a unit sphere $S^{n+p}$. One of the interesting questions in the geometry of the minimal submanifolds of $S^{n+p}$ is to obtain conditions under which they are totally geodesic. These conditions generally involve the pinching of the sectional curvatures, Ricci curvatures, or the scalar curvature (or equivalently the square of the length of the second fundamental form). In [3] for $n \geq 4$, it is proved that if the Ricci curvatures of a minimal submanifold in $S^{n+p}$ are greater than $n-2$, then $M$ is totally geodesic, where $n=\operatorname{dim} M$. For $n=3$ also the above result holds and is proved in [6]. As a natural generalization of this result one may expect that if the scalar curvature $S$ of an $n$-dimensional compact minimal submanifold of $S^{n+p}$ satisfies $S>n(n-2)$, then $M$ is totally geodesic. This will improve all the existing results involving pinching of the scalar curvature for the minimal submanifolds of $S^{n+p}$, and specially 
that in [2]. However, it is not known whether this result holds even in dimension 3. Recently in [6] a slightly courser (than expected above) result is proved in dimension 3, namely there it is shown that for a 3-dimensional compact minimal submanifold $M$ of $S^{3+p}$, the condition $S>4$ implies that $M$ is totally geodesic.

In this paper we study $n$-dimensional compact minimal submanifolds in $S^{n+p}$ with scalar curvature satisfying the pinching condition $S>n(n-2)$. We show that for $p \leq 2$ these submanifolds are totally geodesic (cf. Theorem 3.2 and Corollary 3.1). However, for codimension $p \geq 2$, we have to put additional restrictions on the curvature tensor corresponding to the normal connection to get the result (cf. Theorem 3.1 and Corollary 4.1). We also show that the scalar curvature $S$ of a non-totally geodesic $n$-dimensional nonnegatively curved minimal submanifold in $S^{n+p}$ with flat normal connection satisfies $n(n-p-1) \leq S \leq n(n-2)$ (cf. Theorem 4.1). Since for a compact hypersurface $M$ of $S^{n+1}$ the normal connection is flat, we use the above estimate for the scalar curvature $S$ of a non-negatively curved minimal hypersurface $M$ in $S^{n+1}$ to infer (cf. Corollary 4.2), that either $M$ is totally geodesic or else it is isometric to the hypersurface $S^{m}\left(\sqrt{\frac{m}{n}}\right) \times S^{n-m}\left(\sqrt{\frac{n-m}{n}}\right)$ considered in $[\mathbf{2}]$.

As a consequence of Corollary 4.2, we conclude that the only non-negatively curved compact minimal hypersurface in $S^{n+1}$ which is diffeomorphic to $S^{n}$ is totally geodesic sphere, giving a result in the direction of a question asked by Yau in [7] (cf. p. 692, Problem 99).

\section{Preliminaries.}

Let $M$ be an $n$-dimensional compact minimal submanifold of the unit sphere $S^{n+p}$. We denote by $g$ the Riemannian metric on $S^{n+p}$ as well as that induced on $M$, and by $\bar{\nabla}$ and $\nabla$ the Riemannian connections $S^{n+p}$ on $M$ and respectively. Then we have

$$
\bar{\nabla}_{X} Y=\nabla_{X} Y+h(X, Y), \quad \bar{\nabla}_{X} N=-A_{N} X+\nabla_{X}^{\perp} Y
$$

$X, Y \in \chi(M), N \in \Gamma(\nu)$, where $h$ is the second fundamental form, $\chi(M)$ is the Lie algebra of smooth vector fields on $M, \Gamma(\nu)$ is the space of smooth sections of the normal bundle $\nu$ of $M, \nabla^{\perp}$ is the normal connection and $A_{N}$ is the Weingarten map corresponding to the normal $N \in \Gamma(\nu)$ which satisfies

$$
g\left(A_{N} X, Y\right)=g(h(X, Y), N), \quad X, Y \in \chi(M), N \in \Gamma(\nu) .
$$


For the submanifold $M$ the equations of Gauss, Codazzi and Ricci are respectively

$$
\begin{aligned}
& R(X, Y) Z=g(Y, Z) X-g(X, Z) Y+A_{h(Y, Z)} X-A_{h(X, Z)} Y \\
& (\nabla h)(X, Y, Z)=(\nabla h)(Y, Z, X) \\
& R^{\perp}\left(X, Y, N_{1}, N_{2}\right)=g\left(\left[A_{N_{I}}, A_{N_{2}}\right](X), Y\right)
\end{aligned}
$$

for $X, Y, Z \in \chi(M), \quad N_{1}, N_{2} \in \Gamma(\nu)$, where $R, R^{\perp}$ are the curvature tensors corresponding to the connections $\nabla$ and $\nabla^{\perp}$ respectively and the covariant derivative $(\nabla h)(X, Y, Z)$ is given by

$$
(\nabla h)(X, Y, Z)=\nabla_{X}^{\perp} h(Y, Z)-h\left(\nabla_{X} Y, Z\right)-h\left(Y, \nabla_{X} Z\right) .
$$

The second covariant derivative $\left(\nabla^{2} h\right)(X, Y, Z, W)$ is defined as

$$
\begin{aligned}
\left(\nabla^{2} h\right)(X, Y, Z, W)= & \nabla_{X}^{\perp}(\nabla h)(Y, Z, W)-(\nabla h)\left(\nabla_{X} Y, Z, W\right) \\
& -(\nabla h)\left(Y, \nabla_{X} Z, W\right)-(\nabla h)\left(Y, Z, \nabla_{X} W\right)
\end{aligned}
$$

and we have the Ricci identity

$$
\begin{aligned}
& \left(\nabla^{2} h\right)(X, Y, Z, W)-\left(\nabla^{2} h\right)(Y, X, Z, W) \\
& =R^{\perp}(X, Y) h(Z, W)-h(R(X, Y) Z, W)-h(Z, R(X, Y) W) .
\end{aligned}
$$

For a local orthonormal frame $\left\{e_{1}, \ldots, e_{n}\right\}$ on $M$, as $M$ is a minimal submanifold we have

$$
\sum_{i} h\left(e_{i}, e_{i}\right)=0, \sum_{i}(\nabla h)\left(X, e_{i}, e_{i}\right)=0, \sum_{i}\left(\nabla^{2} h\right)\left(X, Y, e_{i}, e_{i}\right)=0 .
$$

Let Ric be the Ricci tensor of $M$. Then the Ricci operator $Q$ is a symmetric operator defined by

$$
\operatorname{Ric}(X, Y)=g(Q(X), Y), X, Y \in \chi(M) .
$$

The Gauss Equation (2.3) gives the following expression for the Ricci operator $Q$ of the minimal submanifold $M$

$$
Q(X)=(n-1) X-\sum_{i} A_{h\left(e_{i}, X\right)} e_{i},
$$

where $\left\{e_{1}, \ldots, e_{n}\right\}$ is a local orthonormal frame on $M$. The scalar curvature $S=\sum_{i} \operatorname{Ric}\left(e_{i}, e_{i}\right)$ of the minimal submanifold is given by

$$
S=n(n-1)-\|h\|^{2},
$$

where $\|h\|^{2}=\sum_{i j}\left\|h\left(e_{i}, e_{j}\right)\right\|^{2}$ is the square of the length of the second fundamental form. 
Lemma 2.1. Let $M$ be an n-dimensional compact minimal submanifold of the unit sphere $S^{n+p}$. Then

$$
\begin{aligned}
\int_{M}\{ & \|\nabla h\|^{2}+\sum_{i j k}\left[R^{\perp}\left(e_{k}, e_{i} ; h\left(e_{j}, e_{k}\right), h\left(e_{i}, e_{j}\right)\right)\right. \\
& \left.\left.-R\left(e_{k}, e_{i} ; e_{j}, A_{h\left(e_{i}, e_{j}\right)} e_{k}\right)\right]+\sum_{i j} \operatorname{Ric}\left(e_{i}, A_{h\left(e_{i}, e_{j}\right)} e_{j}\right)\right\} d v=0 .
\end{aligned}
$$

Proof. Define $f: M \rightarrow R$ by $f=\frac{1}{2}\|h\|^{2}$. Then the Laplacian of $f$ given by

$$
\Delta f=\sum_{k}\left[e_{k} e_{k}(f)-\nabla_{e_{k}} e_{k}(f)\right],
$$

can be shown to satisfy

$$
\Delta f=\sum_{i j k} g\left(\left(\nabla^{2} h\right)\left(e_{k}, e_{k}, e_{i}, e_{j}\right), h\left(e_{i}, e_{j}\right)\right)+\sum_{i j k}\left\|(\nabla h)\left(e_{i}, e_{j}, e_{k}\right)\right\|^{2} .
$$

Now, using the Ricci identity (2.6) and Equations (2.4), (2.7) in the above equation and integrating the resulting equation we get the integral formula (2.10).

Next we define $\|R\|^{2}$ the square of the length of the curvature tensor field of $M$ by

$$
\|R\|^{2}=\sum_{i j k}\left\|R\left(e_{i}, e_{j}\right) e_{k}\right\|^{2}
$$

where $\left\{e_{1}, \ldots, e_{n}\right\}$ is a local orthonormal frame on $M$. Then using Equations (2.3) and (2.7) we immediately get:

Lemma 2.2. Let $M$ be an n-dimensional compact minimal submanifold of the unit sphere $S^{n+p}$. Then

$$
\begin{aligned}
\|R\|^{2}= & 2 n(n-1)-4\|h\|^{2}+2\left\|A_{h}\right\|^{2} \\
& -2 \sum_{i j k} g\left(A_{h\left(e_{j}, e_{k}\right)} e_{i}, A_{h\left(e_{i}, e_{k}\right)} e_{j}\right)
\end{aligned}
$$

where $\left\|A_{h}\right\|^{2}=\sum_{i j k}\left\|A_{h\left(e_{i}, e_{j}\right)} e_{k}\right\|^{2}$ and $\left\{e_{1}, \ldots, e_{n}\right\}$ is a local orthonormal frame on $M$.

Lemma 2.3. Let $M$ be an n-dimensional compact minimal submanifold of the unit sphere $S^{n+p}$. Then

$$
\sum_{i j k} R\left(e_{k}, e_{i} ; e_{j}, A_{h\left(e_{i}, e_{j}\right)} e_{k}\right)=\frac{1}{2}\|R\|^{2}-S,
$$

where $S$ is the scalar curvature of $M$ and $\left\{e_{1}, \ldots, e_{n}\right\}$ is a local orthonormal frame on $M$. 
Proof. Equation (2.3) implies

$$
A_{h\left(e_{i}, e_{j}\right)} e_{k}=R\left(e_{k}, e_{i}\right) e_{j}+A_{h\left(e_{k}, e_{j}\right)} e_{i}-\delta_{i j} e_{k}+\delta_{k j} e_{i} .
$$

Taking inner product with $A_{h\left(e_{i}, e_{j}\right)} e_{k}$ in the above equation and noticing that

$$
\sum_{i j k} R\left(e_{k}, e_{i} ; e_{j}, A_{h\left(e_{i}, e_{j}\right)} e_{k}\right)=-\sum_{i j k} R\left(e_{k}, e_{i} ; e_{j}, A_{h\left(e_{k}, e_{j}\right)} e_{i}\right)
$$

we get (2.12).

\section{Minimal submanifolds with pinched scalar curvature.}

Let $M$ be an $n$-dimensional compact minimal submanifold of the unit sphere $S^{n+p}$. We choose a local orthonormal frames $\left\{e_{1}, \ldots, e_{n}\right\}$ on $M$ and $\left\{N_{1}, \ldots\right.$, $\left.N_{p}\right\}$ that of normals and define the function $K^{\perp}: M \rightarrow R$ by

$$
K^{\perp}=\sum_{i j \alpha \beta}\left[R^{\perp}\left(e_{i}, e_{j}, N_{\alpha}, N_{\beta}\right]^{2}\right.
$$

and call it the normal curvature of the minimal submanifold. We also define a function $\varphi: M \rightarrow R$ by

$$
\varphi=2 \sum_{\alpha<\beta}\left\|A_{\alpha}\right\|^{2}\left\|A_{\beta}\right\|^{2},
$$

where $A_{\alpha}=A_{N_{\alpha}}$ is the Weingarten map in the direction of the normal vector $N_{\alpha}$.

Theorem 3.1. Let $M$ be an n-dimensional compact minimal submanifold of the unit sphere $S^{n+p}$. If the scalar curvature $S$ and the normal curvature $K^{\perp}$ of $M$ satisfy $S>n(n-2)$ and $K^{\perp} \leq \varphi$ respectively, then $M$ is totally geodesic.

Proof. Let $\left\{e_{1}, \ldots, e_{n}\right\}$ be a local orthonormal frame on $M$. Then Equations (2.3) and (2.8) imply

$$
\begin{gathered}
A_{h\left(e_{j}, e_{k}\right)} e_{i}=R\left(e_{i}, e_{k}\right) e_{j}-\delta_{k j} e_{i}+\delta_{i j} e_{k}+A_{h\left(e_{i}, e_{j}\right)} e_{k} . \\
\sum_{k} A_{h\left(e_{j}, e_{k}\right)} e_{k}=(n-1) e_{j}-Q\left(e_{j}\right) .
\end{gathered}
$$

Taking inner product in (3.1) with $A_{h\left(e_{i}, e_{j}\right)} e_{k}$, we arrive at

$$
\begin{aligned}
& \sum_{i j k} g\left(A_{h\left(e_{i}, e_{j}\right)} e_{k}, A_{h\left(e_{j}, e_{k}\right)} e_{i}\right) \\
& =\left\|A_{h}\right\|^{2}+\sum_{i j k} R\left(e_{i}, e_{k} ; e_{j}, A_{h\left(e_{i}, e_{j}\right)} e_{k}\right)-\|h\|^{2} .
\end{aligned}
$$


Similarly Equation (3.2) gives

$$
\begin{aligned}
\sum_{i j k} g\left(A_{h\left(e_{j}, e_{k}\right)} e_{k}, A_{h\left(e_{i}, e_{j}\right)} e_{i}\right)=(n-1) & \|h\|^{2} \\
& -\sum_{i j} \operatorname{Ric}\left(e_{j}, A_{h\left(e_{i}, e_{j}\right)} e_{i}\right) .
\end{aligned}
$$

Now, using Equations (3.3) and (3.4) in (2.5) we arrive at

$$
\begin{aligned}
R^{\perp}\left(e_{k}, e_{i}, h\left(e_{i}, e_{j}\right), h\left(e_{j}, e_{k}\right)\right)= & \sum_{i j k} R\left(e_{i}, e_{k} ; e_{j}, A_{h\left(e_{i}, e_{j}\right)} e_{k}\right) \\
& +\sum_{i j} \operatorname{Ric}\left(e_{j}, A_{h\left(e_{i}, e_{j}\right)} e_{i}\right) \\
& +\left\|A_{h}\right\|^{2}-n\|h\|^{2} .
\end{aligned}
$$

For a local orthonormal frame $\left\{N_{1}, \ldots, N_{p}\right\}$ of normals we have

$$
\begin{aligned}
\sum_{i j} \operatorname{Ric}\left(e_{j}, A_{h\left(e_{i}, e_{j}\right)} e_{i}\right) & =\sum_{\alpha i j} g\left(A_{\alpha} e_{i}, e_{j}\right) \operatorname{Ric}\left(e_{j}, A_{\alpha} e_{i}\right) \\
& =\sum_{\alpha i} g\left(A_{\alpha} e_{i}, e_{j}\right) \operatorname{Ric}\left(\sum_{j} g\left(A_{\alpha} e_{i}, e_{j}\right) e_{j}, A_{\alpha} e_{i}\right) \\
& =\sum_{\alpha i} \operatorname{Ric}\left(A_{\alpha} e_{i}, A_{\alpha} e_{i}\right) .
\end{aligned}
$$

Now, using (2.8) in (3.6) we arrive at

$$
\begin{aligned}
\sum_{i j} \operatorname{Ric}\left(e_{j}, A_{h\left(e_{i}, e_{j}\right)} e_{i}\right) & =(n-1) \sum_{\alpha i}\left\|A_{\alpha} e_{i}\right\|^{2}-\sum_{\alpha i j}\left\|h\left(e_{j}, A_{\alpha} e_{i}\right)\right\|^{2} \\
& =(n-1)\|h\|^{2}-\sum_{\alpha \beta}\left\|A_{\alpha} A_{\beta}\right\|^{2} .
\end{aligned}
$$

Also we observe that

$$
\sum_{i j k} g\left(A_{h\left(e_{j}, e_{k}\right)} e_{i}, A_{h\left(e_{i}, e_{k}\right)} e_{j}\right)=\sum_{i j \alpha \beta} g\left(A_{\alpha} e_{i}, A_{\beta} e_{j}\right) g\left(A_{\alpha} e_{j}, A_{\beta} e_{i}\right)
$$

and

$$
\begin{aligned}
\sum_{\alpha \beta}\left\|A_{\alpha} A_{\beta}-A_{\beta} A_{\alpha}\right\|^{2} & =\sum_{i j \alpha \beta} g\left(\left(A_{\alpha} A_{\beta}-A_{\beta} A_{\alpha}\right)\left(e_{i}\right), e_{j}\right)^{2} \\
& =-2 \sum_{i j \alpha \beta} g\left(A_{\alpha} e_{i}, A_{\beta} e_{j}\right) g\left(A_{\alpha} e_{j}, A_{\beta} e_{i}\right) \\
& +2 \sum_{\alpha \beta}\left\|A_{\alpha} A_{\beta}\right\|^{2}
\end{aligned}
$$


where we have used $\left\|A_{\alpha} A_{\beta}\right\|^{2}=\left\|A_{\beta} A_{\alpha}\right\|^{2}$ which follows from the fact that $A_{\alpha}$ and $A_{\beta}$ are symmetric. We use Equations (3.8) and (3.9) in (2.11) to arrive at

$$
\begin{aligned}
\|R\|^{2}=2 n(n-1)-4\|h\|^{2}+ & 2\left\|A_{h}\right\|^{2} \\
& +\sum_{\alpha \beta}\left\|A_{\alpha} A_{\beta}-A_{\beta} A_{\alpha}\right\|^{2}-2 \sum_{\alpha \beta}\left\|A_{\alpha} A_{\beta}\right\|^{2}
\end{aligned}
$$

and consequently Lemma 2.3 gives

$\begin{aligned} 2 \sum_{i j k} R\left(e_{k}, e_{i} ; e_{j}, A_{h\left(e_{i}, e_{j}\right)} e_{k}\right) & =2 n(n-1)-4\|h\|^{2}+2\left\|A_{h}\right\|^{2} \\ & (3.10) \\ & -2 S+\sum_{\alpha \beta}\left\|A_{\alpha} A_{\beta}-A_{\beta} A_{\alpha}\right\|^{2}-2 \sum_{\alpha \beta}\left\|A_{\alpha} A_{\beta}\right\|^{2} .\end{aligned}$

The integral formula (2.10) in view of (3.5) takes the form

$$
\begin{array}{r}
\int_{M}\left\{\|\nabla h\|^{2}+\left\|A_{h}\right\|^{2}-n\|h\|^{2}-2 \sum_{i j k} R\left(e_{k}, e_{i} ; e_{j}, A_{h\left(e_{i}, e_{j}\right)} e_{k}\right)\right. \\
\left.+2 \sum_{i j} \operatorname{Ric}\left(e_{j}, A_{h\left(e_{i}, e_{j}\right)} e_{i}\right)\right\} d v=0 .
\end{array}
$$

Thus using (3.7), (3.10) and (2.9) in (3.11), we conclude

$$
\int_{M}\left\{\|\nabla h\|^{2}+n\|h\|^{2}-\left\|A_{h}\right\|^{2}-\sum_{\alpha \beta}\left\|A_{\alpha} A_{\beta}-A_{\beta} A_{\alpha}\right\|^{2}\right\} d v=0 .
$$

Note that in view of Equation (2.5) we have

$$
\begin{aligned}
K^{\perp} & =\sum_{i j \alpha \beta}\left[R^{\perp}\left(e_{i}, e_{j}, N_{\alpha}, N_{\beta}\right)\right]^{2}=\sum_{i j \alpha \beta} g\left(\left[A_{\alpha}, A_{\beta}\right]\left(e_{i}\right), e_{j}\right)^{2} \\
& =\sum_{\alpha \beta}\left[\sum_{i j} g\left(\left(A_{\alpha} A_{\beta}-A_{\beta} A_{\alpha}\right)\left(e_{i}\right), e_{j}\right)^{2}\right] \\
& =\sum_{\alpha \beta}\left\|A_{\alpha} A_{\beta}-A_{\beta} A_{\alpha}\right\|^{2} .
\end{aligned}
$$


Also we have

$$
\begin{aligned}
\left\|A_{h}\right\|^{2} & =\sum_{i j k}\left\|A_{h\left(e_{i}, e_{j}\right)} e_{k}\right\|^{2}=\sum_{i j k \alpha} g\left(A_{\alpha} e_{i}, e_{j}\right)^{2}\left\|A_{\alpha} e_{k}\right\|^{2} \\
& =\sum_{i j \alpha}\left\|A_{\alpha}\right\|^{2} g\left(A_{\alpha} e_{i}, e_{j}\right)^{2}=\sum_{\alpha}\left\|A_{\alpha}\right\|^{4} \\
& =\left(\sum_{\alpha}\left\|A_{\alpha}\right\|^{2}\right)^{2}-2 \sum_{\alpha<\beta}\left\|A_{\alpha}\right\|^{2}\left\|A_{\beta}\right\|^{2}
\end{aligned}
$$

which gives

$$
\left\|A_{h}\right\|^{2}=\|h\|^{4}-2 \sum_{\alpha<\beta}\left\|A_{\alpha}\right\|^{2}\left\|A_{\beta}\right\|^{2} .
$$

Using Equations (3.13) and (3.14) in (3.12), we arrive at

$$
\int_{M}\left\{\|\nabla h\|^{2}+\left(n-\|h\|^{2}\right)\|h\|^{2}+\left(\varphi-K^{\perp}\right)\right\} d v=0 .
$$

Now using $S>n(n-2)$ with $(2.4)$, we conclude that $\|h\|^{2}<n$ and hence the integral formula (3.15) implies that $M$ is totally geodesic.

Theorem 3.2. Let $M$ be an n-dimensional compact minimal submanifold of the unit sphere $S^{n+2}$. If the scalar curvature $S$ of $M$ satisfies $S>n(n-2)$, then $M$ is totally geodesic.

Proof. In codimension 2 the integral formula (3.12) together with (3.14) gives

$\int_{M}\left\{\|\nabla h\|^{2}+\left(n-\|h\|^{2}\right)\|h\|^{2}+2\left\|A_{1}\right\|^{2}\left\|A_{2}\right\|^{2}-\left\|A_{1} A_{2}-A_{2} A_{1}\right\|^{2}\right\} d v=0$,

where $\left\{N_{1}, N_{2}\right\}$ is a local orthonormal frame of normals on $M$ and $A_{\alpha}=$ $A_{N_{\alpha}}, \alpha=1,2$. Then using Lemma 1 in ([2], p. 64), and the hypothesis $S>n(n-2)$, we get from above integral formula that $\|h\|^{2}=0$, that is $M$ is totally geodesic.

Remark 3.1. For Veronese surface $M$ in $S^{4}$, one can show that the normal curvature $K^{\perp}$ and the function $\varphi$ appearing in Theorem 3.1 satisfy $K^{\perp}=$ $\varphi=\frac{8}{9}$. Indeed we can choose a local orthonormal frame $\left\{e_{1}, e_{2}\right\}$ on $M$ and a local orthonormal frame $\left\{N_{1}, N_{2}\right\}$ of normals such that the Weingarten maps $A_{N_{1}}, A_{N_{2}}$ take the form (cf. [2])

$$
A_{1}=\left(\begin{array}{cc}
0 & 1 \\
1 & 0
\end{array}\right), \quad A_{2}=\left(\begin{array}{cc}
1 & 0 \\
0 & -1
\end{array}\right)
$$


Remark 3.2. For a compact minimal hypersurface $M$ in $S^{n+1}$ we have $K^{\perp}=0$ and $\varphi=0$ and consequently the integral formula (3.15) takes the form

$$
\int_{M}\left\{\|\nabla A\|^{2}+\left(n-\|A\|^{2}\right)\|A\|^{2}\right\} d v=0
$$

where $A$ is the Weingarten map of the hypersurface. Thus we have:

Corollary 3.1. Let $M$ be an $n$-dimensional compact minimal hypersurface of the unit sphere $S^{n+1}$. If the scalar curvature $S$ of $M$ satisfies $S \geq n(n-2)$, then $M$ is either totally geodesic or else is isometric to the hypersurface $S^{m}\left(\sqrt{\frac{m}{n}}\right) \times S^{n-m}\left(\sqrt{\frac{n-m}{n}}\right)$.

Proof. If $M$ is not totally geodesic, then the above integral formula gives that the Weingarten map $A$ is parallel and $\|A\|^{2}=n$, consequently as in [2], $M$ is isometric to the hypersurface $S^{m}\left(\sqrt{\frac{m}{n}}\right) \times S^{n-m}\left(\sqrt{\frac{n-m}{n}}\right)$ of $S^{n+1}$.

\section{Minimal submanifolds with flat normal connection.}

In this section we study compact minimal submanifolds of the unit sphere $S^{n+p}$ which have flat normal connection, that is $R^{\perp}=0$. First, we prove the following theorem which estimates the scalar curvature of such submanifolds which are non-totally geodesic and non-negatively curved.

Theorem 4.1. Let $M$ be an n-dimensional compact non-negatively curved minimal submanifold of the unit sphere $S^{n+p}$ with flat normal connection. Then either $M$ is totally geodesic or else the scalar curvature $S$ of $M$ satisfies $n(n-p-1) \leq S \leq n(n-2)$.

Proof. Since the normal connection is flat we have $R^{\perp}=0$ and that all the Weingarten maps $A_{N}, N \in \Gamma(\nu)$, can be diagonalized with respect to the same local orthonormal frame $\left\{e_{1}, \ldots, e_{n}\right\}$ (cf. [1], p. 127). Choose a local orthonormal frame $\left\{N_{1}, \ldots, N_{p}\right\}$ of normals such that $A_{\alpha}\left(e_{i}\right)=\lambda_{i}^{\alpha} e_{i}$, where 
$\lambda_{i}^{\alpha}$ are the smooth functions and $\alpha=1, \ldots, p$. Then we have

$$
\begin{aligned}
& \sum_{i j} \operatorname{Ric}\left(e_{j}, A_{h\left(e_{i}, e_{j}\right)} e_{j}\right)-\sum_{i j k} R\left(e_{k}, e_{i} ; e_{j}, A_{h\left(e_{i}, e_{j}\right)} e_{k}\right) \\
& =\sum_{\alpha}\left[\sum_{i j} g\left(A_{\alpha} e_{i}, e_{j}\right) \operatorname{Ric}\left(e_{i}, A_{\alpha} e_{j}\right)-\sum_{i j k} g\left(A_{\alpha} e_{i}, e_{j}\right) R\left(e_{k}, e_{i} ; e_{j}, A_{\alpha} e_{k}\right)\right] \\
& =\sum_{\alpha}\left[\sum_{j} \operatorname{Ric}\left(A_{\alpha} e_{j}, A_{\alpha} e_{j}\right)-\sum_{j k} R\left(e_{k}, A_{\alpha} e_{j} ; e_{j}, A_{\alpha} e_{k}\right)\right] \\
& =\sum_{\alpha}\left[\sum_{j k} R\left(e_{k}, A_{\alpha} e_{j} ; A_{\alpha} e_{j}, e_{k}\right)-\sum_{j k} R\left(e_{k}, A_{\alpha} e_{j} ; e_{j}, A_{\alpha} e_{k}\right)\right] \\
& =\sum_{\alpha j k}\left(\left(\lambda_{j}^{\alpha}\right)^{2} K_{k j}-\lambda_{j}^{\alpha} \lambda_{k}^{\alpha} K_{k j}\right)
\end{aligned}
$$

where $K_{k j}=R\left(e_{k}, e_{j} ; e_{j}, e_{k}\right)$ is the sectional curvature of the plane section spanned by $\left\{e_{k}, e_{j}\right\}$. Consequently we have

$$
\begin{aligned}
& \sum_{i j} \operatorname{Ric}\left(e_{i}, A_{h\left(e_{i}, e_{j}\right)} e_{j}\right)-\sum_{i j k} R\left(e_{k}, e_{i} ; e_{j}, A_{h\left(e_{i}, e_{j}\right)} e_{k}\right) \\
& =\frac{1}{2}\left[\sum_{\alpha j k} 2\left(\lambda_{j}^{\alpha}\right)^{2} K_{k j}-2 \sum_{\alpha j k} \lambda_{j}^{\alpha} \lambda_{k}^{\alpha} K_{k j}\right] \\
& =\frac{1}{2}\left[\sum_{\alpha j k}\left(\lambda_{j}^{\alpha}\right)^{2} K_{k j}+\sum_{\alpha j k}\left(\lambda_{k}^{\alpha}\right)^{2} K_{k j}-2 \sum_{\alpha j k} \lambda_{j}^{\alpha} \lambda_{k}^{\alpha} K_{k j}\right] \\
& =\frac{1}{2}\left[\sum_{\alpha j k}\left(\lambda_{j}^{\alpha}-\lambda_{k}^{\alpha}\right)^{2} K_{k j}\right] \geq 0 .
\end{aligned}
$$

Then the integral formula (2.10) with $R^{\perp}=0$ and (4.1) gives

(4.2) $\|\nabla h\|=0$,

$$
\sum_{i j} \operatorname{Ric}\left(e_{i}, A_{h\left(e_{i}, e_{j}\right)} e_{j}\right)-\sum_{i j k} R\left(e_{k}, e_{i} ; e_{j}, A_{h\left(e_{i}, e_{j}\right)} e_{k}\right)=0 .
$$

Now Equation (3.5) with $R^{\perp}=0$ and the second equation in (4.2) yields

$$
\left\|A_{h}\right\|^{2}-n\|h\|^{2}=0 .
$$


Also, we have

$$
\begin{aligned}
\left\|A_{h}\right\|^{2}=\sum_{\alpha}\left\|A_{\alpha}\right\|^{4} & =\left(\sum_{\alpha}\left\|A_{\alpha}\right\|^{2}\right)^{2}-2 \sum_{\alpha<\beta}\left\|A_{\alpha}\right\|^{2}\left\|A_{\beta}\right\|^{2} \\
& =\|h\|^{4}-2 \sum_{\alpha<\beta}\left\|A_{\alpha}\right\|^{2}\left\|A_{\beta}\right\|^{2} .
\end{aligned}
$$

Combining (4.3) and (4.4), we arrive at

$$
\|h\|^{2}\left(\|h\|^{2}-n\right) \geq 0 .
$$

Also from (4.3), we have $\sum_{\alpha}\left(\left\|A_{\alpha}\right\|^{4}-n\left\|A_{\alpha}\right\|^{2}\right)=0$, or equivalently

$$
\sum_{\alpha}\left(\left\|A_{\alpha}\right\|^{2}-\frac{n}{2}\right)^{2}=\frac{n^{2} p}{4} .
$$

Now using Schwarz inequality, we get

$$
\sum_{\alpha}\left(\left\|A_{\alpha}\right\|^{2}-\frac{n}{2}\right)^{2} \geq \frac{1}{p}\left[\sum_{\alpha}\left(\left\|A_{\alpha}\right\|^{2}-\frac{n}{2}\right)\right]^{2}=\frac{1}{p}\left(\|h\|^{2}-\frac{n p}{2}\right)^{2} .
$$

Combining (4.6) and (4.7), we conclude that

$$
\|h\|^{2}\left(\|h\|^{2}-n p\right) \leq 0 \text {. }
$$

This proves that either $M$ is totally geodesic or $\|h\|^{2} \leq n p$. If $M$ is not totally geodesic, then from $\|h\|^{2} \leq n p$ together with (4.5) we get $n \leq\|h\|^{2} \leq$ $n p$, which by Equation (2.9) is equivalent to $n(n-p-1) \leq S \leq n(n-2)$.

Next we prove the following:

Theorem 4.2. Let $M$ be an n-dimensional compact minimal submanifold of the unit sphere $S^{n+p}$ with flat normal connection. If the scalar curvature $S$ of $M$ satisfies $S \geq n(n-2)$, then $M$ is either totally geodesic or is isometric to the hypersurface $S^{m}\left(\sqrt{\frac{m}{n}}\right) \times S^{n-m}\left(\sqrt{\frac{n-m}{n}}\right)$.

Proof. Since $R^{\perp}=0$, Equation (3.5) gives

$$
\begin{aligned}
& \sum_{i j} \operatorname{Ric}\left(e_{i}, A_{h\left(e_{i}, e_{j}\right)} e_{j}\right)-\sum_{i j k} R\left(e_{k}, e_{i} ; e_{j}, A_{h\left(e_{i}, e_{j}\right)} e_{k}\right) \\
&=n\|h\|^{2}-\left\|A_{h}\right\|^{2} .
\end{aligned}
$$

Thus the integral formula (2.10) in view of (4.9) and $R^{\perp}=0$, takes the form

$$
\int_{M}\left\{\|\nabla h\|^{2}+n\|h\|^{2}-\left\|A_{h}\right\|^{2}\right\} d v=0 .
$$


For a local orthonormal frame $\left\{N_{1}, \ldots, N_{p}\right\}$ of normals, using the expression for $\left\|A_{h}\right\|^{2}$ as given in (4.4) into (4.10) we arrive at

$$
\int_{M}\left\{\|\nabla h\|^{2}+\left(n-\|h\|^{2}\right)\|h\|^{2}+2 \sum_{\alpha<\beta}\left\|A_{\alpha}\right\|^{2}\left\|A_{\beta}\right\|^{2}\right\} d v=0 .
$$

Since $S \geq n(n-2)$, that is $\|h\|^{2} \leq n$, above integral gives $\|\nabla h\|=0$, $\|h\|^{2}\left(n-\|h\|^{2}\right)=0, \sum_{\alpha<\beta}\left\|A_{\alpha}\right\|^{2}\left\|A_{\beta}\right\|^{2}=0$. The last two equations give either $M$ is totally geodesic or else $\|h\|^{2}=n$ and $A_{\beta}=0, \beta=2, \ldots, p$. In the second case, we have as in [2] that $M$ is isometric to the hypersurface $S^{m}\left(\sqrt{\frac{m}{n}}\right) \times S^{n-m}\left(\sqrt{\frac{n-m}{n}}\right)$ of $S^{n+1}$.

Now as applications of these theorems, first we have:

Corollary 4.1. Let $M$ be an n-dimensional compact minimal submanifold of the unit sphere $S^{n+p}$ with flat normal connection. If the scalar curvature $S$ of $M$ satisfies $S>n(n-2)$, then $M$ is totally geodesic.

Corollary 4.2. Let $M$ be a non-negatively curved compact minimal hypersurface of $S^{n+1}$. Then $M$ is either totally geodesic or is isometric to the hypersurface $S^{m}\left(\sqrt{\frac{m}{n}}\right) \times S^{n-m}\left(\sqrt{\frac{n-m}{n}}\right)$.

Proof. Since $M$ is hypersurface, the normal connection is flat, by Theorem 4.1, we have either $M$ is totally geodesic or the scalar curvature is given by $S=n(n-2)$. If $M$ is non-totally geodesic, we have $\|h\|^{2}=n$ and consequently by the result in [2], $M$ is isometric to the hypersurface $S^{m}\left(\sqrt{\frac{m}{n}}\right) \times S^{n-m}\left(\sqrt{\frac{n-m}{n}}\right)$.

Remark 4.1. In [2], it is asked that the values of $\|h\|^{2}$ should determine the compact hypersurfaces up to rigid motion in the ambient sphere $S^{n+1}$ (cf. p. 75). The above Corollary shows that for non-negatively curved hypersurfaces in $S^{n+1},\|h\|^{2}$ takes only two values 0 and $n$ and the corresponding hypersurfaces are known. Thus the Corollary 4.2 can be thought of as a result in the direction of this question.

We also have the following consequence of Corollary 4.2:

Corollary 4.3. Let $M$ be a non-negatively curved compact minimal hypersurface of $S^{n+1}$. If $M$ is diffeomorphic to $S^{n}$, then $M$ is totally geodesic.

Remark 4.2. One of the important questions concerning the hypersurfaces of $S^{n+1}$ is to show that "the only embedded minimal hypersurface in $S^{n+1}$ which is diffeomorphic to $S^{n}$ is the totally geodesic sphere" (cf. [7], p. 692, Problem-99). Corollary 4.3 can be considered as a result in this direction for the class of non-negatively curved minimal hypersurfaces in $S^{n+1}$. 
Finally, we prove:

Theorem 4.3. Let $M$ be an n-dimensional compact minimal submanifold of the unit sphere $S^{n+p}$ with flat normal connection. If $M$ is of constant sectional curvature $c \geq 0$, then either $M$ is flat $(c=0)$ and $p \geq(n-1)$, or totally geodesic.

Proof. Let $\left\{e_{1}, \ldots, e_{n}\right\}$ be a local orthonormal frame on $M$. Then for the minimal submanifold $M$ of constant sectional curvature $c$, Equation (2.3) can be restated as

$$
(c-1)\left(\delta_{j k} e_{i}-\delta_{i k} e_{j}\right)=A_{h\left(e_{j}, e_{k}\right)} e_{i}-A_{h\left(e_{i}, e_{k}\right)} e_{j} .
$$

Taking inner product with $A_{h\left(e_{j}, e_{k}\right)} e_{i}$ and summing the resulting equation we arrive at

$$
(1-c)\|h\|^{2}=\left\|A_{h}\right\|^{2}-\sum_{i j k} g\left(A_{h\left(e_{i}, e_{k}\right)} e_{j}, A_{h\left(e_{j}, e_{k}\right)} e_{i}\right) .
$$

Now as the normal connection is flat, $R^{\perp}=0$ and with the help of Equation (2.5) we compute

$$
\begin{aligned}
\sum_{i j k} g\left(A_{h\left(e_{i}, e_{k}\right)} e_{j}, A_{h\left(e_{j}, e_{k}\right)} e_{i}\right) & =\sum_{i j k} g\left(A_{h\left(e_{j}, e_{k}\right)} e_{j}, A_{h\left(e_{i}, e_{k}\right)} e_{i}\right) \\
& =\sum_{i k} g\left(\sum_{j} A_{h\left(e_{j}, e_{k}\right)} e_{j}, A_{h\left(e_{i}, e_{k}\right)} e_{i}\right) \\
& =\sum_{i k} g\left((n-1) e_{k}-Q\left(e_{k}\right), A_{h\left(e_{i}, e_{k}\right)} e_{i}\right) \\
& =(n-1)\|h\|^{2}-\sum_{i k} \operatorname{Ric}\left(e_{k}, A_{h\left(e_{i}, e_{k}\right)} e_{i}\right) .
\end{aligned}
$$

However for $M$ the Ricci curvature satisfies Ric $(X, Y)=(n-1) c g(X, Y)$ and consequently,

$$
\sum_{i j k} g\left(A_{h\left(e_{i}, e_{k}\right)} e_{j}, A_{h\left(e_{j}, e_{k}\right)} e_{i}\right)=(n-1)(1-c)\|h\|^{2} .
$$

Using this equation in (4.12), we get

$$
\left\|A_{h}\right\|^{2}=n(1-c)\|h\|^{2} .
$$

Note that Equation (4.3) holds for non-negatively curved compact minimal submanifolds of $S^{n+p}$ with flat normal connection. Thus combining (4.3) and (4.13), we conclude that $c\|h\|^{2}=0$ and this proves that either $c=0$ or $M$ is totally geodesic. However if $c=0$, then $S=0$ and consequently by Thorem 4.1, $n(n-p-1) \leq 0$ (as $M$ is non-totally geodesic), which implies $p \geq n-1$. 
Corollary 4.4. There is no compact flat minimal hypersurface in $S^{4}$.

\section{References}

[1] B.Y. Chen, Total Mean Curvature and Submanifolds of Finite Type, World Scientific, 1983.

[2] S.S. Chern, M. DoCarmo and S. Kobayashi, Minimal submanifolds of a sphere with second fundamental form of constant length, Functional Analysis and Related Topics, Springer Verlag, (1970), 59-75.

[3] N. Ejiri, Compact minimal submanifolds of a sphere with positive Ricci curvature, J. Math. Soc. Japan, 31 (1979), 251-256.

[4] K. Moshimo, Minimal immersions of 3-dimensional sphere in to spheres, Osaka J. Math., 21 (1984), 721-732.

[5] J. Simon, Minimal varieties in Riemannian manifolds, Ann. of Math., 88(2) (1968), 62-105.

[6] Y.B. Shen, Curvature pinching for three-dimensional minimal submanifolds in a sphere, Proc. Amer. Math. Soc., 115(3) (1992), 791-795.

[7] S.T. Yau, Problem section, Seminar on Differential Geometry, Princeton Univ. Press, 1982.

Received July 15, 1998.

King Saud University

RIYADH-11451

SAudi Arabia

E-mail address: shariefd@ksu.edu.sa 